\title{
L'harmonisation des tarifications dans les compagnies de chemins de fer
}

Maurice Wolkowitsch

\section{(2) OpenEdition}

\section{Journals}

Édition électronique

URL : https://journals.openedition.org/rhcf/1940

DOI : 10.4000/rhcf.1940

\section{Éditeur}

Rails \& histoire

\section{Édition imprimée}

Date de publication : 1 février 2003

Pagination : 68-97

ISBN : 0996-9403

ISSN : 0996-9403

\section{Référence électronique}

Maurice Wolkowitsch, "L'harmonisation des tarifications dans les compagnies de chemins de fer », Revue d'histoire des chemins de fer [En ligne], 26 | 2003, mis en ligne le 19 janvier 2015, consulté le 22 avril 2022. URL : http://journals.openedition.org/rhcf/1940 ; DOI : https://doi.org/10.4000/rhcf.1940 


\section{Maurice WOLKOWITSCH}

\section{L'harmonisation des tarifications dans les compagnies de chemins de fer}

L'étude des chemins de fer secondaires conduit à l'examen des choix de leurs dirigeants et des élus départementaux à l'origine de leur construction. La recherche de normes communes n'est pas leur souci ; le mot standardisation ne caractérise pas leur matériel ${ }^{1}$. Peut-on parler d'accords, au moins de convergences dans le domaine tarifaire? Tel est l'objet de notre intervention. Nous avons examiné la situation en $1912^{2}$.

Un chemin de fer secondaire ne se définit pas par le seul écartement des voies : les chemins de fer d'intérêt local (CFIL) du Nord allient une ligne à voie normale à un réseau à voie étroite; le réseau à voie étroite de Sud France est en partie d'intérêt général. Dans notre optique, nous considérons comme compagnies secondaires celles qui ne correspondent pas aux 7 grands réseaux ${ }^{3}$, ni aux Ceintures de Paris.

Notre démarche débute par un rappel des règles communes aux grands réseaux, puis à toutes les compagnies ferroviaires. Nous présentons ensuite les tarifications voyageurs, puis marchandises, propres aux compagnies secondaires. Enfin l'analyse des tarifs communs permet d'apprécier si des efforts réels ont été entrepris en vue d'une harmonisation tarifaire commune à toutes les compagnies ferroviaires. Auparavant, il convient de souligner le rôle primordial des chemins de fer dans la vie sociale et économique au début $\mathrm{du} \mathrm{xx}^{\mathrm{e}}$ siècle ; la puissance de l'automobile tend à le faire oublier.

\section{Le rôle du chemin de fer}

Jusqu'au début des années vingt, le rôle du chemin de fer est incontesté, même si en 1911 quelques lignes routières sont subventionnées. Hommes et marchandises, au-delà de quelques kilomètres, circulent par les voies ferrées normales ou étroites. Les lignes secondaires et

1- Pascal Puig, «Le Meusien : utilisation d'une voie métrique par l’Armée française », in « Armées et chemins de fer », Revue d'histoire des chemins de fer, $\mathrm{n}^{\circ} 15$ (automne 1996), p. 88-116.

2- Recueil général des tarifs des chemins de fer et leurs correspondances - Grande Vitesse, Paris, Chaix, 1912, 999 p.

3- Le rachat de l'Ouest par l'État en 1908 a ramené ce nombre à six. Mais les homologations de tarifs intervenues avant cette date au titre de l'Ouest figurent sous ce nom dans les documents tarifaires. Pour la période ultérieure, on trouve indifféremment les mentions Ouest, État ou Ouest-État. 
celles du plan Freycinet répondent aux besoins locaux et permettent aussi l'accès à une gare de bifurcation sur une grande ligne. Ouvriers, curistes, émigrants, étudiants, paysans, notables, voyageurs de commerce peuplent les voitures. Wagons et fourgons transportent matériaux de construction, dentelles, cercueils, billets de banque, engrais, actions, chiens, or, voitures. Une tarification correspond à chaque élément du trafic. Le trafic postal bénéficie d'emplacements réservés sur la moindre ligne. Des chercheurs ont condamné a posteriori ces lignes, taxées d'électoralistes, oubliant l'absence d'alternative au chemin de fer lors de leur construction. Les positions maximalistes ${ }^{4}$ se font écho alors que l'analyse sereine de P. Vidal de Lablache mérite davantage d'être citée : "C'est qu'en effet à mesure que se sont déroulées les conséquences du chemin de fer, la différence entre les contrées qui en sont pourvues et celles qui ne l'étaient pas a créé une telle infériorité pour ces dernières qu'à tout prix il a fallu la combattre. ${ }^{5} \gg$ Ces voies irriguant tout le territoire ouvrent de nouveaux marchés, favorisent l'évolution agricole des Dombes ${ }^{6}$ ou des terres granitiques grâce à l'apport des engrais et amendements. Ce maillage ferroviaire dense, même si l'évolution technique le condamnait à terme, a permis à la France d'ignorer dans ses montagnes et campagnes reculées la misère qu'offrent encore tant de terres de l'Europe méditerranéenne et orientale. Ces évolutions positives sont liées à des tarifications adaptées à des demandes locales.

\section{Règles communes aux grandes compagnies et à toutes les compagnies ferroviaires}

Un accord de 1883 entre le ministère des Travaux publics et les grandes compagnies est une première étape vers plus de cohérence dans la tarification et ses conditions d'application. Les mesures seront progressives et n'élimineront pas l'esprit de concurrence. Les grands réseaux établissent des tarifs généraux communs et simplifient leurs tarifs ; lois, décrets, ordonnances, arrêtés ministériels sanctionnent ces accords conduisant à l'homologation. Cette réglementation tatillonne

4- «Le développement du réseau ferré d'intérêt général a pour conséquence la transformation des routes et chemins départementaux et [leur] conversion en CFIL... » (Rapport Thévenet, ingénieur en chef, Archives départementales de la Dordogne, Série S Chemins de fer économiques 1879-1884). En 1930, l'ingénieur De Conninck demande : «le déferrage de toutes les voies ferrées pour y établir des routes.»

5- Pierre Vidal de Lablache, Principes de géographie humaine, Paris, Colin, 1921, 327 p., p. 241.

6- Pierre Protat, «Compagnie des Dombes », étude dactyl. déposée à l'AHICF, 44 p. +44 p. d'Annexes. 
n'impose pas une harmonisation parfaite en tolérant des exceptions : encore en 1912, le minimum de perception correspondant à $6 \mathrm{~km}$ est réduit à 3 sur le PLM ; il correspond sur l'État à une somme de $0,05 \mathrm{~F}$ et non à la taxation d'une distance minimale ; sur ce réseau les militaires, mais aussi les voyageurs de $1^{\text {te }}$ classe, bénéficient de tarifs plus avantageux; le prix de transport des boîtes à lait vides en retour varie d'un réseau à l'autre : franco en G.V. sur l'Est, franco en G.V. mais soumis aux frais d'enregistrement sur le PLM et le PO, franco en P.V. sur l'Ouest mais avec les frais d'enregistrement.

Les barèmes des prix de transport au vk (voyageur/kilomètre) et à la tk (tonne/kilomètre) sont fournis pour chaque élément du trafic : bagages, messageries et marchandises, denrées avec la liste des 41 produits concernés, chiens, marchandises ne pesant pas $200 \mathrm{~kg}$ sous $1 \mathrm{~m}^{3}$ de volume, marchandises vénéneuses, dangereuses ou explosives, masses indivisibles ou de dimensions exceptionnelles, animaux en cage ou panier (cobaye, chevreau, caille...) pesant $30 \mathrm{~kg}$ maximum (animal + contenant) taxés en messagerie, voitures hippomobiles à une ou deux banquettes, voitures automobiles, pompes funèbres, produits taxés ad valorem (finances, valeurs, objets d'art, broderies, dentelles...), animaux taxés par tête de bétail, animaux dangereux ou valant plus de $5000 \mathrm{~F}$. La classification des marchandises est progressivement unifiée. Le principe de la taxation par l'itinéraire le plus court est adopté, avec son corollaire, l'acheminement par la compagnie qui l'offre. Les frais accessoires sont énoncés avec la même minutie : enregistrements, pesage, manutention, magasinage avec délais d'enlèvement, dépôt de bagages avec les cas particuliers (piano, voiture d'enfant, brouette, bicyclette, automobile, machines-outils, arbres et arbustes). Les délais de livraison sont définis et accompagnés des horaires d'ouverture des gares avec la liste de celles livrant jour et nuit le lait et les denrées périssables et, pour quelques villes desservies par le PLM, les fleurs coupées.

Certaines règles sont communes à toutes les compagnies ferroviaires principales et secondaires : réduction accordée aux enfants et militaires - cependant Sud France en exclut les officiers sur son réseau des Alpes-Maritimes - délivrance de billets aller-retour, de cartes d'abonnement, de billets collectifs, définition des produits de valeur comme objets de plus de $300 \mathrm{~F}$ au $\mathrm{kg}$, conditionnement des produits financiers, expédition en G.V. par le premier train de voyageurs assurant ces transports trois heures après le dépôt de la marchandise, délai de transmission entre réseaux dans les gares de transit (trois heures dans les gares communes, six heures en cas de gares distinctes reliées par le rail), égalité de traitement entre les clients demandant le transport de masses 
indivisibles au-delà d'un certain poids ${ }^{7}$. Le droit d'enregistrement est fixé sur tous les réseaux à $0,10 \mathrm{~F}$; il est perçu une seule fois pour l'emprunt des lignes de plusieurs grands réseaux; mais il est perçu pour chaque emprunt de lignes de réseaux secondaires, sauf exception : une expédition entre deux gares situées sur des réseaux secondaires différents en transit par un grand réseau paye trois droits d'enregistrement.

\section{La tarification sur les réseaux secondaires}

La situation au sein des compagnies secondaires est complexe. Toute généralisation est hasardeuse, c'est le domaine du particularisme et de l'exception. Chaque compagnie est dans un contexte socioéconomique spécifique ; elle entretient avec un, voire deux grands réseaux des rapports plus ou moins étroits sur le plan administratif comme sur celui des conditions de transmission des marchandises dans les gares de transit.

Les compagnies secondaires établissent leur tarification par référence aux tarifs et conditions d'application des grands réseaux, soit pour les adopter, soit pour définir des exceptions. Elles font souvent précéder leur document tarifaire de la formule : "Les conditions d'application sont les mêmes que sur les grands réseaux avec les exceptions suivantes : [...]», suit la liste des trafics pour lesquels les tarifs de base ou les conditions d'application diffèrent de ceux des grands réseaux. Pour faciliter les comparaisons, nous nous appuierons sur les taxes en francs au vk (voyageur-kilomètre) ou à la tk (tonne-kilomètre). Ces données varient d'une compagnie à l'autre, parfois pour diverses lignes d'une même compagnie. Nous avons déjà relevé des failles dans la réglementation commune aux grands réseaux; l'entente entre compagnies secondaires était plus difficile, vu leur attachement à leurs propres pratiques et les dispositions de leurs cahiers des charges.

\section{Les tarifs voyageurs}

Le prix varie en fonction du confort : les compagnies secondaires conservent les caractéristiques des grands réseaux : «voiture de $1^{\text {re }}$ classe couverte, garnie, fermée à glaces, $2^{\mathrm{e}}$ classe couverte, banquettes rembourrées, fermée à glaces, $3^{\mathrm{e}}$ classe couverte, fermée à vitres. » Les réseaux secondaires se satisfont souvent de deux classes : la $1^{\text {te }}$ correspond à la $1^{\text {te }}$ des grands réseaux, la $2^{\mathrm{e}}$ dispose de l'équipement de la $3^{\mathrm{e}}$.

7- Si un tel transport est effectué pour un client il doit être assuré pour tous les clients en faisant la demande dans les trois mois suivant le premier acheminement. Sur les grands réseaux, le tarif est majoré de $50 \%$ entre 3 et 5 t, doublé entre 5 et $8 \mathrm{t}$. 
En fait, les compagnies font payer le prix du confort offert par le matériel en service : sur divers secteurs d'exploitation, la S.G.C.F.E. fait payer un même prix pour des classes différentes (tabl. 1); sur les trois réseaux voisins de l'Allier, du Cher et de la Nièvre, la tarification diffère : le $\mathrm{km}$ en $1^{\text {re }}$ classe coûte entre 0,07725 et 0,103 . Dans le cadre plus étroit des Chemins de fer départementaux des Ardennes, on enregistre presque un tarif différent pour chaque ligne : 4 en $1^{\text {te }}$ classe compris entre 0,08 et $0,103,5$ en $2^{\mathrm{e}}$ classe entre 0,055 et 0,075 . L'analyse du prix du vk sur plus de 30 réseaux signale une différence de l'ordre de $45 \%$ pour chaque classe ; l'amplitude serait plus élevée, en prenant en compte les lignes de montagne : le tarif est de 0,226 en $1^{\text {te }}$ classe et 0,16995 en $2^{\mathrm{e}}$ classe entre Pierrefitte-Nestalas et Cauterets. Les grands réseaux offrent en $3^{\mathrm{e}}$ classe un tarif inférieur à celui des compagnies secondaires, du même ordre en $1^{\text {te }}$ et $2^{\mathrm{e}}$ classe (annexe 1).

\begin{tabular}{|l|c|c|c|c|c|c|}
\hline Tarif en F & \multicolumn{2}{|c|}{0,077725} & \multicolumn{2}{c|}{0,0618} & \multicolumn{2}{c|}{0,05665} \\
\hline Classe & $1^{\text {1e }}$ & $2^{\mathrm{e}}$ & $2^{\mathrm{e}}$ & $3^{\mathrm{e}}$ & $2^{\mathrm{e}}$ & $3^{\mathrm{e}}$ \\
\hline Réseau & Cher & Gironde & Nièvre & $\begin{array}{c}\text { Somme et } \\
\text { lignes de } \\
\text { Bussy à } \\
\text { Ercheu et } \\
\text { Aumale à } \\
\text { Envermeu }\end{array}$ & Allier & \\
\hline & Nord & & & & Cher & \\
\hline & & & & & Nord & \\
\hline
\end{tabular}

Tableau 1. Prix du km/voyageur sur les réseaux et lignes de la SGCFE, 1912.

Les grands réseaux délivrent des cartes d'abonnements ordinaires, de famille ${ }^{8}$ ou d'associés valables sur tous ou un ou deux d'entre eux pour un parcours ou une zone; les compagnies secondaires sont exclues de cet accord, de même que leurs lignes ne peuvent figurer sur un billet circulaire à itinéraire facultatif; chacune délivre le même type de cartes d'abonnement (annexe 2) valables sur leur seul réseau, ce qui

8- Le mot « famille » est compris dans un sens très large chaque fois qu'il est employé dans la tarification : mari, femme, grands-parents, beaux-parents, enfants, gendres, belles-filles, beaux-frères, belle-sœurs, oncles, tantes, neveux, nièces, précepteurs, gouvernantes, serviteurs attachés à la famille, chauffeur si une automobile est acheminée par train. 
en limite l'intérêt ; le voyageur empruntant les lignes des grands réseaux et d'une compagnie secondaire achète deux cartes d'abonnement. Les distances modestes parcourues sur les réseaux secondaires ne permettent pas l'application de barèmes dégressifs efficaces.

Les compagnies pratiquent une politique commerciale visant à développer le trafic: les grands réseaux offrent des aller-retour avec une réduction de $25 \%$ par rapport à l'achat de deux allers simples, la validité est fonction de la distance. Les compagnies secondaires s'inspirent d'une même pratique ; chacune adopte sa propre réglementation; quelques-unes conservent les tarifs des grands réseaux. Les aller-retour sont proposés pour des parcours entre deux gares du réseau ou de façon plus restrictive d'une même ligne : les usagers bénéficient de réductions de $25 \%$ pour les Chemins de fer du Centre, $20 \%$ pour le Tramway des Deux-Sèvres, $10 \%$ pour les CFIL du Morbihan... Des conditions encore plus complexes régissent les tarifs aller-retour sur certaines compagnies. La Compagnie du chemin de fer de La Mure propose des réductions de $25 \%$ en $1^{\text {re }}, 28 \%$ en $2^{\mathrm{e}}$ classe et $36 \%$ en $3^{\mathrm{e}}$ pour tout aller-retour entre deux gares de la ligne. Les CFIL de l'Hérault délivrent des aller-retour avec réduction de $30 \%$ les dimanches et fêtes, $15 \%$ en semaine sur le prix de deux allers simples ; mais, en semaine, la réduction est aussi de $30 \%$ au départ de Montpellier pour toutes les gares des lignes Montpellier à Rabieux et à Saint-Chinian et d'Agde à Font-Mars, également pour les voyageurs accomplissant plus de $40 \mathrm{~km}$ entre des gares de ces lignes. On s'explique mal cette complexité. Les Chemins de fer départementaux d'Ardèche et HauteLoire ont un tarif spécial pour les ouvriers et ouvrières des manufactures et des exploitations agricoles rentrant dans leur famille du samedi au dimanche. Les Chemins de fer de Limagne sur des relations bien définies autour de Gerzat et Saint-Bauzire, ceux de Côte-d'Or offrent des réductions de $50 \%$ sur le prix des abonnements pour les déplacements quotidiens des ouvriers se rendant dans leur atelier. De très nombreuses compagnies accordent le même avantage aux professeurs des deux sexes, instituteurs et institutrices, élèves, étudiants, apprentis et jeunes ouvriers jusqu'à 26 ans ou 27 si le service militaire est accompli. Le minimum de perception de $6 \mathrm{~km}$ est ramené à $3 \mathrm{~km}$ pour les voyageurs des Tramways de l'Indre qui parcourent moins de $3 \mathrm{~km}$, mesure destinée à capter le trafic urbain et périurbain entre Châteauroux et Déols ; les tramways de la Côte-d'Or pratiquent une même politique entre DijonVille et Dijon-Porte neuve. A Grasse, le minimum de perception est abaissé à $1 \mathrm{~km}$ pour les voyageurs allant de la gare PLM à l'hôtel de ville. On perçoit les efforts entrepris par les compagnies secondaires 
pour offrir des tarifs réduits à tous ceux dont l'activité exige de se déplacer régulièrement. Elles n'ont pas négligé pour autant une autre clientèle.

Des tarifs sont mis en place pour les groupes, mais l'isolement des compagnies réduit les déplacements dans l'espace. Des conditions sont exigées : une distance minimale $(12 \mathrm{~km}$ pour les Chemins de fer économiques du Nord), un nombre minimum de participants (25 pour le chemin de fer d'Andelot à Levier), la nature des groupes concernés variant suivant les compagnies est définie avec un luxe de détails surprenant. Les déplacements liés à l'enseignement ou au tourisme font l'objet d'une attention particulière (annexe 4). Dans un même esprit, les compagnies mettent en service des trains spéciaux, louent des wagonssalons dans la mesure où elles en disposent et où elles peuvent les acheminer par des convois réguliers. Les CFIL de l'Hérault proposent des places de luxe entre Montpellier et Palavas avec un supplément de $50 \%$ sur le tarif de $1^{\text {re }}$.

Si nous retrouvons dans les tarifs marchandises le même souci de prendre en compte les réalités locales, nous voyons aussi les compagnies secondaires confrontées à des problèmes liés aux capacités de leur matériel.

\section{Tarifs marchandises}

Les compagnies secondaires, pour le transport des marchandises ne pesant pas $200 \mathrm{~kg}$ sous le volume de $1 \mathrm{~m}^{3}$, imposent « une majoration de $50 \%$ des prix fixés au tarif général, les expéditions de moins de 40 $\mathrm{kg}$ et les marchandises nommément énoncées au cahier des charges en étant exonérées ». Ce faisant, elles suivent à la lettre la réglementation des grandes compagnies: tel est le cas pour les Chemins de fer du Beaujolais ou les Chemins de fer départementaux de la Mayenne. D'autres pratiquent une augmentation de $100 \%$ : Chemins de fer économiques des Charentes, Tramway de Challans à Fromentine.

Des règles particulières aux compagnies secondaires sont liées à la nature et à l'hétérogénéité de leurs équipements (annexe 3).

Les compagnies secondaires transportent, sauf exception, les masses indivisibles jusqu'à 5 t. Les conditions d'application varient en fonction des capacités du matériel et des infrastructures et de l'équipement des gares en engins de levage. Beaucoup de compagnies n'ont pas la capacité d'acheminer des charges aussi lourdes. Les tarifications fixées sont très diverses. Au-delà de $3 \mathrm{t}$, la clientèle a la charge et la responsabilité des manutentions et le prix de transport est fixé par l'administration sur proposition de la compagnie. 
Des problèmes similaires se posent pour l'acheminement d'objets de dimensions exceptionnelles, dépassant celles du matériel. En cas de dérogation, on retrouve les mêmes règles que pour les excès de poids. Toutes les compagnies n'offrent pas les mêmes possibilités qui sont déterminées par celles des matériels en service. Une même compagnie avec un même écartement peut sur ses lignes ne pas imposer les mêmes limites, si les matériels en service sont différents (Sud France).

Un second facteur d'originalité tarifaire pour les compagnies secondaires tient à leur volonté de répondre aux besoins locaux

Aucune surprise à trouver des tarifs spéciaux pour le transport des huitres sur diverses lignes secondaires du littoral vendéen (réduction de $25 \%$ ), pour celui du lait sur celles de la Compagnie des Charentes ${ }^{9}$, pour celui des citrons, feuilles de menthe, jasmin rose et géranium sur celles de Sud France, pour celui des grenouilles sur le Chemin de fer de Marlieux à Châtillon. Les Chemins de fer départementaux des Bouches-duRhône portent un intérêt légitime au trafic des fruits, légumes et primeurs, offrant un tarif de $0,28 \mathrm{~F}$ la th à confronter au $0,50 \mathrm{~F}$ fixé au tarif général des messageries, soit une réduction de $44 \%$. Un tarif exceptionnel encore plus avantageux était prévu pour les expéditions par $50 \mathrm{~kg}$ entre Lamanon d'une part, Arles et Meyrargues d'autre part.

La réutilisation des emballages vides est la règle au début du $\mathrm{xx}^{\mathrm{e}}$ siècle ; leur retour à vide à l'expéditeur est indispensable ; les compagnies grandes et petites répondent à cette demande sous condition d'une expédition préalable à plein en G.V. en sens inverse sur une même relation. Nous avons évoqué la question à propos du lait acheminé par les grands réseaux vers toutes les villes. Des tarifs très bas étaient consentis pour les boites, paniers, caisses, sacs, toiles. Les Chemins de fer départementaux des Bouches-du-Rhône offraient une tarification avantageuse entre les gares de transit avec le PLM et celles des zones de production (tabl. 2) ; un tarif encore plus bas est accordé pour des relations privilégiées de Barbentane à Châteaurenard et de Tarascon à Saint-Rémy-de-Provence, de l'ordre de 0,04 F par tonne. Sud France dispose d'un tarif pour le transport des cocons frais, mais inévitablement pour le retour des emballages dans lesquels ils voyagent ${ }^{10}$.

9- Le tarif spécial s'applique aux expéditions d'au moins 50 litres, lait et boîte pesés ensemble, le tarif est de 0,25 la tk retour franco des boîtes vides. Au tarif général des messageries, en fonction du poids, le prix serait compris entre 0,45 et 0,56 F la tk.

10- Les cocons frais sont transportés au tarif de 0,2435 la tk, les emballages vides (suite page suivante) 


\begin{tabular}{|c|c|c|}
\hline & 0 à $25 \mathrm{~kg}$ & plus de $25 \mathrm{~kg}$ \\
\hline 1 à $15 \mathrm{~km}$ & 0,25 & $\begin{array}{c}0,25 \text { pour les premiers } 25 \mathrm{~kg}+0,05 \mathrm{par} \\
\text { fraction indivisible de } 10 \mathrm{~kg} \text { à partir du } \\
26^{\mathrm{e}}\end{array}$ \\
\hline Plus de $15 \mathrm{~km}$ & 0,25 & $\begin{array}{c}0,25 \text { pour les premiers } 25 \mathrm{~kg}+0,10 \mathrm{par} \\
\text { fraction indivisible de } 10 \mathrm{~kg} \text { à partir du } \\
26^{\mathrm{e}}\end{array}$ \\
\hline
\end{tabular}

Tableau 2. Tarif de retour des emballages vides de fruits, légumes et primeurs sur les Chemins de fer départementaux des Bouches-du-Rhône en F par tk, 1912.

\section{Ententes tarifaires entre compagnies secondaires}

Tous ces tarifs marchandises ou voyageurs s'appliquent dans le cadre de chaque compagnie secondaire. Nous relevons cependant quelques tentatives de rapprochements entre compagnies secondaires, freinées par l'absence de continuité physique de leur ligne ou réseau.

Cinq compagnies adoptent les mêmes exceptions par rapport aux tarifs généraux G.V. des grands réseaux ; elles le précisent par un renvoi à la tarification d'une même compagnie secondaire, les Chemins de fer du Finistère ; malgré cette affirmation, une lecture attentive montre que, sur un point précis, l'une ou l'autre fixe encore des conditions spécifiques. Ces compagnies ne dépendent pas d'une même autorité de tutelle, leur direction d'exploitation est sur le terrain à Brest, Quimper, Laval, Chartres, Avranches et Poitiers, mais elles partagent le même siège social, 3, rue Beldant, au Mans. Cette unité de lieu traduit sans doute des similitudes dans les conseils d'administration qui auraient poussé vers des tarifications voisines. Un exemple de même nature apparait dans le Nord et le Pas-de-Calais (annexe 5).

Trois réseaux en continuité peuvent aller plus loin dans cette politique ; eux aussi partagent le même siège social, 54 boulevard Haussmann, à Paris, la direction de l'exploitation étant à Bordeaux : Chemin de fer du Born et du Marensin et de la ligne Soustons à Léon font les mêmes exceptions aux tarifs des grandes compagnies que la Compagnie de chemin de fer des Landes. Les transports de gare à gare (suite)

en retour pour $0,138 \mathrm{~F}$. L'application du tarif général pour les messageries serait de 0,45 jusqu'à $40 \mathrm{~kg}, 0,36$ pour plus de $40 \mathrm{~kg}$, la majoration de $50 \%$ pour marchandises de moins de $200 \mathrm{~kg}$ sous un volume de $1 \mathrm{~m}^{3}$ ne s'applique pas. La compagnie dégage sa responsabilité en cas d'éclosion des cocons en cours de voyage. 
de chacun des réseaux sont considérés comme effectués sur un seul et même réseau, en conséquence les prix des tarifs généraux et spéciaux sont appliqués sur le parcours total d'après la distance accomplie. Cette unité se traduit par exemple par la possibilité avec une seule carte d'abonnement à demi-tarif d'obtenir la réduction sur les trois réseaux et d'établir des barèmes dégressifs avec la distance plus avantageux pour les marchandises ${ }^{11}$.

Ces essais d'ententes entre compagnies secondaires restent timides. Vont-elles être associées aux nombreux tarifs communs aux grands réseaux et se dégager du cercle étroit de leur activité en ouvrant à leur clientèle plus de facilités pour accéder à de larges horizons ? Telle est la question à laquelle il convient de répondre.

\section{Les tarifs communs}

En 1912 les grandes compagnies offrent de nombreux tarifs communs visant tous les trafics voyageurs et marchandises entre gares et même stations de chacun d'eux; les compagnies secondaires ne sont pas associées à ces larges ententes. Sauf exception, une compagnie secondaire participe à un tarif commun avec un grand réseau ; les cahiers des charges des compagnies secondaires sont peut-être un frein à des accords qui ne seraient pas compatibles avec leurs obligations.

\section{Tarifs communs voyageurs}

Les tarifs communs ont pour objectif le développement du trafic; mais les compagnies ont toujours le souci de conserver le voyageur sur leurs lignes; elles rencontrent des difficultés à se plier à des règles communes. Deux exemples illustrent cet état d'esprit. La délivrance des billets de séjours touristiques commence suivant les réseaux le jeudi ou le vendredi avant les Rameaux. Les voyageurs de $2^{\mathrm{e}}$ classe sur les grands réseaux sont transportés sur les chemins de fer secondaires ne disposant pas de trois classes parfois en $2^{\mathrm{e}}$ classe, le plus souvent en $1^{\text {te }}$; certaines compagnies laissent le choix aux voyageurs, sous condition de payer le prix correspondant à la classe choisie.

Les tarifs communs simples prévoient la délivrance de billets directs, aller ou aller-retour dont le prix est calculé : «...par la soudure des tarifs intérieurs généraux ou spéciaux propres à chaque compagnie. »

11- Signalons que les Chemins de fer des Pyrénées-Orientales ont le même siège social que les compagnies citées, appliquent les tarifs des grands réseaux sauf pour les militaires, marins et leurs chevaux et les pompes funèbres générales où le renvoi est aux tarifs des Chemins de fer des Landes. La direction de l'exploitation est à Perpignan. 
Cette commodité n'entraîne aucun avantage tarifaire ; si l'addition des distances parcourues sur chaque réseau permet de franchir des seuils définis, la validité d'un AR peut être accrue. La délivrance de ces billets (tarif spécial G.V. 2 de chaque compagnie) concerne généralement un nombre précis de relations ; ainsi État, Chemins de fer départementaux des Charentes et des Deux-Sèvres ont à trois un tarif commun : des AR sont délivrés pour 30 gares des compagnies secondaires au départ de Niort, 28 de La Rochelle, 20 de Rochefort, 12 de Bordeaux (État ou Saint-Jean), 11 de Saintes, 5 de Melle, 2 de Brioux; pour chaque relation la gare de transit autorisée avec l'État est indiquée. Des conditions d'application encore plus restrictives et complexes apparaissent dans les tarifs communs État-Chemins de fer de l'Anjou ou Orléans-Chemins de fer départementaux.

Dans plusieurs cas, outre la facilité matérielle d'avoir à prendre un seul billet, le voyageur peut trouver des avantages. Modestes dans le tarif commun Nord-Chemin de fer de Somain à Anzin où un seul droit d'enregistrement est perçu par la gare expéditrice, plus intéressants dans les tarifs communs prévoyant la liberté de choix entre itinéraires concurrents, celui par le grand réseau étant souvent le plus long. Le voyageur bénéficie de plus de services et souvent d'un prix forfaitaire fixé quel que soit l'itinéraire choisi (tabl. 3). Les voyageurs isolés bénéficient rarement d'avantages substantiels. Un tarif commun PLM-Chemin de fer de La Mure est une exception. 45 gares du PLM situées dans le périmètre Lyon -Valence - Marseille - Veynes - Grenoble - Aix-les-Bains délivrent des AR valables quatre jours pour les gares du Chemin de fer de La Mure avec des réductions de $25 \%$ en $1{ }^{\text {re }}, 20 \%$ en $2^{\mathrm{e}}$ et $3^{\mathrm{e}}$; la compagnie secondaire applique les mêmes réductions, mais adopte une validité de 2 jours pour $50 \mathrm{~km}, 3$ pour 100, plus 1 par tranche de $100 \mathrm{~km}$. Un cas original est fourni par un tarif commun à cinq : Orléans, Ouest, État, Tramways de la Sarthe, Chemin de fer de Mamers à Saint-Calais; toutes les gares de ces réseaux, situées dans la Sarthe, délivrent des cartes d'abonnement permettant la délivrance de billets à demi-tarif ; la répartition des recettes est fixée en fonction de la part de chaque réseau dans le kilométrage total de voies ferrées du département.

Dans la région parisienne, les grands réseaux et les Ceintures ont établi de multiples tarifs communs, notamment des abonnements pour les déplacements quotidiens avec des réductions, en plus, de $50 \%$ pour les scolaires et apprentis. Dans un seul cas, une compagnie secondaire est associée : le tarif commun Ouest-Nord-Chemin de fer d'Enghien à Montmorency prévoit des AR à prix fixes et des abonnements entre 
toutes les gares de la compagnie secondaire et vice versa et Paris-Nord, Ouest, Pontoise, Argenteuil, Saint-Denis... Les compagnies secondaires mettent souvent en circulation des trains les jours de foires et marchés mais nous n'avons relevé qu'un cas de tarif commun destiné à en favoriser l'accès : Midi, Chemins de fer du département des Landes et de Soustons à Léon délivrent des $\mathrm{AR}$ en $3^{\mathrm{e}}$ classe à prix exceptionnels valables un jour, le samedi, jour du marché de Dax au départ de 16 gares des deux compagnies secondaires ; aucune réduction n'est accordée aux enfants ou aux militaires, aucune franchise pour les bagages.

\begin{tabular}{|l|l|l|}
\hline $\begin{array}{l}\text { Relation et vice } \\
\text { versa }\end{array}$ & \multicolumn{1}{|c|}{$\begin{array}{c}\text { Réseaux } \\
\text { concernés }\end{array}$} & Itinéraires et conditions d'application \\
\hline $\begin{array}{l}\text { Rennes à } \\
\text { Fougères }\end{array}$ & $\begin{array}{l}\text { Ouest, Tramways } \\
\text { d'Ille-et-Vilaine }\end{array}$ & $\begin{array}{l}\text { via Vitré, 73km* } \\
\text { via Saint-Aubin-du-Cormier } 53 \mathrm{~km}^{*}\end{array}$ \\
\hline Bordeaux à Blaye & $\begin{array}{l}\text { État } \\
\text { État-Blayais }\end{array}$ & $\begin{array}{l}\text { via Saint-Mariens Saint-Yzan } 70 \mathrm{~km} \\
\text { via Saint-André-de-Cubzac } 49 \mathrm{~km}\end{array}$ \\
\hline $\begin{array}{l}\text { Béziers à } \\
\text { Pézenas }\end{array}$ & $\begin{array}{l}\text { Midi } \\
\text { CFIL Hérault }\end{array}$ & $\begin{array}{l}\text { via Vias } 36 \mathrm{~km} \\
\text { via Servian } 26 \mathrm{~km}\end{array}$ \\
\hline Le Mans à Loué & $\begin{array}{l}\text { Ouest } \\
\text { Tramways de la } \\
\text { Sarthe }\end{array}$ & $\begin{array}{l}\text { via Sillé-le Guillaume } 61 \mathrm{~km}^{* *} \\
\text { via Coulans } 36 \mathrm{~km}{ }^{* *}\end{array}$ \\
\hline $\begin{array}{l}\text { Nantes à } \\
\text { St-Nazaire }\end{array}$ & Orléans & via Savenay $64 \mathrm{~km}$ \\
\hline $\begin{array}{l}\text { St-Nazaire à } \\
\text { Nantes }\end{array}$ & $\begin{array}{l}\text { Bac de Mindin } \\
\text { CFIL Morbihan-État }\end{array}$ & via Paimbœuf $76 \mathrm{~km}$ ou via Pornic $90 \mathrm{~km}$ \\
\hline
\end{tabular}

* Délivrance AR valable 3 jours et uniquement pour le parcours total.

** D'autres gares desservies par les Tramways de la Sarthe sont concernées.

Tableau 3. Liberté de choix entre itinéraires concurrents laissée aux voyageurs dans le cas d'ententes tarifaires entre réseaux, 1912.

Nous avons relevé les tarifs spéciaux propres aux compagnies secondaires pour les pèlerins et les émigrants ; les grands réseaux ont multiplié les tarifs communs entre eux en faveur de ces catégories, sans associer les compagnies secondaires qui rabattent cependant les clients jusqu'aux gares de transit ou comme la Compagnie du Médoc, qui conduit de Bordeaux à Pauillac les émigrants arrivés par les voies de 
l'Orléans ou du Midi, soit en transit à travers la France, soit en provenance de Rodez, Albi, Toulouse, Montauban, Agen et Marmande considérées comme desservant des zones d'émigration.

Les déplacements touristiques sont l'objet d'une attention particulière des grands réseaux. Leurs voies desservent des stations comme Cannes ou Arcachon, ils ont construit des antennes pour en gagner d'autres comme Vichy, mais ils n'atteignent pas toutes les zones littorales, pas plus que le cœur des montagnes. L'élaboration de tarifs communs associant toutes les catégories de compagnies ferroviaires est indispensable. Les textes montrent toujours la volonté des grandes compagnies de garder le plus possible les clients sur leur réseau, de les enfermer dans un carcan d'obligations : durée de validité des billets, saison de délivrance, distance minimale à parcourir, parfois itinéraire défini... On peut distinguer des tarifs destinés aux touristes qui souhaitent visiter des régions en circulant constamment, d'autres conçus pour faciliter l'accès aux 231 stations littorales et 134 villes thermales fréquentées par les baigneurs et curistes et que les compagnies veulent desservir.

Dans la première catégorie, l'accord entre État et Tramway de la Vendée propose la formule originale d'une carte d'excursion de 15 jours donnant libre circulation sur certaines lignes de chaque réseau dans une zone définie $\left(34 \mathrm{~F}\right.$ en $1^{\mathrm{re}}, 28 \mathrm{~F}$ en $2^{\mathrm{e}}, 18 \mathrm{~F}$ en $\left.3^{\mathrm{e}}\right)$. Un tarif État, Chemin de fer économique des Charentes, compagnie de navigation desservant les îles de Ré, Aix et Oléron, accorde aux voyageurs ralliant ou quittant une gare du circuit d'excursion une réduction de $40 \%$ sur le prix de l'aller au départ de toutes les gares, stations et haltes de l'État. Sur les billets circulaires à itinéraires proposés par les compagnies, la participation d'un réseau secondaire s'impose : le PLM a ainsi traité avec le Tramway de Thônes à Annecy, le Funiculaire de Grasse et Sud France pour le parcours de Grasse à Nice par Colomars. En dehors des très nombreux circuits organisés, le PLM offre des voyages circulaires à coupons combinables, pour lesquels les lignes secondaires offrent des parcours attractifs : 149 liaisons, hors des voies du PLM, sont proposées aux voyageurs : 84 par des chemins de fer secondaires, 34 en voitures automobiles, 29 en voitures hippomobiles, un en bateau et même un à dos de mulet ; 22 liaisons sont alpines, 19 provençales, les autres dans le Massif central et en Bourgogne. Sud France est sollicitée pour une série de parcours sur les lignes de Nice à Meyrargues et Toulon à Hyères et Saint-Raphaël, mais aussi le Chemin de fer de la Mure, celui de Voiron à Saint-Béron, des lignes dans le Vercors et autour de Vizille, le chemin de fer du Montenvers. Des clauses rigoureuses doivent être respectées : $300 \mathrm{~km}$ doivent être parcourus sur le PLM, le parcours hors PLM ne 
doit pas dépasser $25 \%$ du kilométrage total. Le prix résulte de l'addition des prix forfaitaires prévus pour chaque parcours hors PLM, tous modes confondus, et du prix pour les parcours sur le PLM suivant un barème comprenant 50 paliers entre 301 et $7000 \mathrm{~km}$. La validité dépend des kilomètres accomplis uniquement sur le PLM (de 30 jours pour $1500 \mathrm{~km}$ à 60 au-delà de $3000 \mathrm{~km}$ ). Les familles de trois personnes bénéficient de $10 \%$ de réduction pour la $3^{\mathrm{e}}, 25 \%$ à partir de la $4^{\mathrm{e}}$.

Le tarif spécial G.V. 106 est destiné à répondre sous quatre chapitres différents aux besoins de ceux qui recherchent des séjours prolongés sur le lieu de leurs vacances. Les chemins de fer secondaires ne sont pas associés à la délivrance des abonnements de bains de mer et de cartes d'excursion donnant libre circulation dans des zones définies. Les grands réseaux multiplient les tarifs communs entre eux pour élargir l'horizon des voyages de loisirs de leurs clientèle, sans se départir totalement de la volonté de la conserver sur leurs lignes. PO et PLM desservant des zones touristiques attractives ont chacun de leur côté un tarif commun avec le Nord, mais non entre eux. Les grandes compagnies associent les compagnies secondaires, lorsque leur réseau ne dessert pas directement une station : un cas sur les 9 tarifs communs pour la délivrance des billets de famille en direction des stations balnéaires et thermales et lieux de vacances ${ }^{12}, 6$ cas sur 16 pour les AR individuels en direction des mêmes stations, valables 33 jours, 10 cas sur 13 pour les AR individuels vers les mêmes stations de validités diverses. Quelques dispositions sont communes pour l'utilisation de ces billets : prolongation de validité à deux reprises, moyennant le versement de $10 \%$ du prix du billet par période de 30 jours, composition de la famille, obligation au départ de Paris d'emprunter le réseau desservant la gare de transit avec le réseau secondaire conduisant à la station... Mais des règlements spécifiques beaucoup plus nombreux régissent l'emploi de ces billets : taux des réductions consenties par les compagnies, contraintes kilométriques conditionnant le bénéfice des avantages tarifaires...

Les compagnies secondaires sont destinées à permettre le parcours entre la gare de transit avec un grand réseau et une station touristique. L'État a réussi à établir des règles communes avec une série

12- Seuls les billets de famille permettent de se rendre ailleurs que dans les stations de séjour ; cela correspond aux déplacements vers les propriétés rurales, châteaux, manoirs et autres ; la conception large de la famille déjà évoquée, incluant les employés, est significative. 
de compagnies secondaires. Des tarifs communs assurant la réciprocité ont été élaborés entre les réseaux du Midi et du Médoc ${ }^{13}$, entre ceux de l'État et des Chemins de fer économiques des Charentes. Plusieurs cas d'association de compagnies de navigation à un tarif commun entre grands réseaux et compagnie secondaire se rencontrent dans les estuaires et pour l'accès aux îles côtières. Une seule compagnie secondaire intérieure délivre des billets de bains de mer pour les stations desservies par l'État (Richelieu à Ligré-Rivière). La volonté des grandes compagnies de conserver le trafic le plus loin possible se traduit dans le choix des gares de transit ; l'exemple de la desserte des côtes varoises illustre cette tendance : elles désignent Hyères ou Saint-Raphaël et non Toulon. La situation est la même en Bretagne.

L'élaboration de tarifs communs pour les voyageurs est réalisée lorsque les grands réseaux jugent en tirer un bénéfice. Un même esprit règne dans le domaine des marchandises.

\section{Les tarifs communs de transport des marchandises}

Les tarifs communs G.V. associant réseaux principaux et secondaires sont rares ; ils sont plus nombreux en P.V. Les modes d'exploitation des compagnies secondaires s'accommodent mal de la notion d'expédition en G.V. La vitesse est réduite, de l'ordre de $25 \mathrm{~km}$ à l'heure, mais cela n'est pas l'essentiel. Plus grave est l'absence de circulations affectées à ce trafic, d'où l'emprunt obligatoire des trains de voyageurs dont la fréquence est faible, souvent deux trains quotidiens. Malgré les règles communes en matière de délai déjà évoquées, les marchandises risquent des attentes prolongées ${ }^{14}$ sur les réseaux secondaires; on atteint des temps de transport où la responsabilité des compagnies peut être engagée ; d'où la méfiance des grands réseaux. L'exemple suivant semble significatif.

Le tarif commun Nord, Chemin de fer de Hermès à Beaumont et Chemin de fer de Méru à La Bosse porte sur le transport de légumes frais, champignons et cressons entre toutes gares de ces réseaux et ParisNord. Par $50 \mathrm{~kg}$ minimum, le prix est de $0,24 \mathrm{~F}$ la tk augmenté de $1,80 \mathrm{~F}$ la t pour manutention et transbordement. La majoration pour marchandise pesant moins de $200 \mathrm{~kg}$ sous $1 \mathrm{~m}^{3}$ de volume ne s'applique pas. Les emballages vides en retour sont acheminés gratuitement en P.V., ou au prix du tarif de l'aller en G.V., calculé sur le poids réel des

13- Les gares de la Compagnie du Médoc délivrent des billets pour Arcachon, celles du Midi pour Soulac-sur-Mer.

14- Un expéditeur apportant une marchandise après le passage du train du matin risque de la voir attendre 8 à 10 heures le train suivant. Une marchandise transmise par un grand réseau à un réseau secondaire après le départ du dernier train de la journée a grande chance de rester une douzaine d'heures en attente. 
emballages par tranches de $10 \mathrm{~kg}$. Sauf cas de force majeure, accident, neige ou verglas, les compagnies abandonnent le tiers du prix de transport pour un retard de $3 \mathrm{~h} 1 / 2$ à 4 h $1 / 2$, les $2 / 3$ entre 4 h $1 / 2$ et $5 \mathrm{~h} 1 / 2$, la totalité au-delà. Les compagnies sont solidairement responsables jusqu'à concurrence du prix de transport; au-delà de 12 heures, l'expéditeur peut exercer un recours et réclamer une indemnisation pour la perte de sa marchandise.

Un tarif commun Midi, Chemin de fer des Landes est établi pour les relations de Dax à deux stations du Chemin de fer des Landes, l'important est « que dans aucun cas, les taxes puissent être supérieures à celles qui résulteraient de l'application des bases du cahier des charges des CFIL du département des Landes ». Sans cette clause explicitée ici, le prix du transport augmenterait pour certaines relations; ce risque n'a-t-il pas conduit des réseaux secondaires à être circonspects avant de s'engager à l'établissement de tarifs communs ?

Deux tarifs communs régissent le transport des raisins. Le premier associe le CFIL de l'Hérault, le PLM, les Ceintures, le Nord et l'Est : il vise le trafic de toutes les gares des lignes CFIL de Montpellier à Palavas, Rabieux et Montbazin à toutes les gares des réseaux cités. Deux barèmes de prix sont fixés : par expédition d'au moins $50 \mathrm{~kg}$, ou par $5 \mathrm{t}$; dans ce cas, une réduction de $10 \%$ du prix de transport est accordée sur la fraction de poids dépassant ce tonnage. Le second tarif destiné à l'exportation associe en plus le réseau de l'Ouest : il s'applique entre toutes les gares des lignes citées du CFIL de l'Hérault et les points frontières avec la Suisse, l'Allemagne, l'Alsace, la Lorraine, le Luxembourg et la Belgique, les ports desservis par le Nord et ceux de Dieppe, le Havre et Rouen. Deux barèmes de prix sont proposés : par expéditions d'au moins $50 \mathrm{~kg}$, par wagon de $5 \mathrm{t}$ réfrigéré appartenant à des particuliers ou à l'administration des chemins de fer et aménagés par les expéditeurs; dans ce cas une réduction de $10 \%$ du prix de transport est consentie sur la totalité du chargement. Le transport de ces aménagements est gratuit à l'aller dans la limite du $1 / 3$ du chargement taxé, gratuit au retour.

Pour des raisons de documentation disponible, nous avons analysé les tarifs communs P.V. $n^{\circ} 102$ à 105 régissant diverses marchandises et 106 traitant des boissons ${ }^{15}$. Nous n'avons pu prendre en compte les transports de pondéreux dont on sait l'importance. Sur 173 tarifs communs étudiés, 40 seulement intègrent une ou deux compagnies secondaires (tabl. 4).

15- Tarifs des Chemins de fer P.V. 102, 50 p. et Tarifs 106, 19 p., Extraits du Recueil général, Paris, Librairie centrale des chemins de fer, janvier 1912. 


\begin{tabular}{|l|c|c|c|}
\hline \multicolumn{1}{|c|}{ Marchandises } & $\begin{array}{c}\text { Nombre } \\
\text { total de } \\
\text { tarifs } \\
\text { communs } \\
\text { tarifs communs } \\
\text { associant des } \\
\text { compagnies } \\
\text { secondaires }\end{array}$ & $\begin{array}{c}\text { Nombre de } \\
\text { compagnies } \\
\text { secondaires } \\
\text { concernées }\end{array}$ \\
\hline $\begin{array}{l}102 \text { - Céréales Farines } \\
\text { Graines Légumes secs } \\
\text { Pâtes alimentaires } \\
\text { Pommes de terre }\end{array}$ & 38 & 10 & 9 \\
\hline $\begin{array}{l}103 \text { - Denrées Fruits } \\
\text { Légumes Produits de } \\
\text { laiterie }\end{array}$ & 36 & 6 & 8 \\
\hline $\begin{array}{l}104 \text { - Eau de mer Sel } \\
\text { Gemme Sel marin }\end{array}$ & 15 & 2 & 2 \\
\hline $\begin{array}{l}105 \text { - Betterave Sucre } \\
\text { Mélasse Dextrine } \\
\text { Glucose Sirop }\end{array}$ & 33 & 12 & 15 \\
\hline 106 - Boissons & 51 & 10 & 9 \\
\hline
\end{tabular}

Tableau 4. Association des compagnies secondaires aux tarifs communs 102 à 106 pour l'année 1912.

Les tarifs communs P.V. offrent certaines conditions d'application identiques : un tarif s'applique entre deux gares ou deux séries de gares désignées, également entre deux gares intermédiaires situées sur l'itinéraire le plus court, à la condition de payer pour le poids et la distance prévus par le règlement; le trafic peut généralement se réaliser dans les deux sens, principe souvent sans objet comme pour le transport de vin ou de produits maraîchers allant évidemment des zones de production vers les marchés de consommation. Les envois peuvent être réalisés en wagons complets ou en expéditions fractionnées, un tonnage minimum étant toujours exigé. Des barèmes dégressifs avec la distance et le poids sont proposés. Ces tarifs offrent aussi des particularités : les délais de transport sont raccourcis ou allongés suivant la nature des marchandises (huîtres ou céréales); la taxation peut reposer sur la capacité des wagons employés et non sur le poids de la marchandise chargée, elle s'exprime en $\mathrm{m}^{3}$ suivant la distance (Nord et réseaux secondaires pour le transport des betteraves); le prix de la tk varie d'une compagnie à l'autre pour une même marchandise et un même palier de distance ; les taxes 
d'enregistrement et les frais de gare sont perçus ou non par chaque compagnie ou par la seule gare expéditrice; les frais de transbordement et transmission aux gares de transit, de manutention au départ et à l'arrivée sont intégrés ou pas dans le prix de transport affiché. Des clauses plus exigeantes figurent, comme le tarif commun réservé aux compagnies secondaires enclavées dans le réseau Nord.

Les compagnies secondaires apparaissent dans les tarifs communs pour le transport des vins, à l'exclusion de toutes les autres boissons. Les trafics partent de certaines zones de production vers les grands marchés de consommation, Paris en priorité. Bourgogne et Champagne sont ignorées. Tous les tarifs prévoient les transports en fûts, dans trois cas en wagons réservoirs ; cette solution prévue avec les Chemins de fer de Camargue à voie étroite exige la mise en wagon réservoir à Arles-Trinquetaille-transit; les réseaux à voie normale des Landes et de l'Hérault évitent ce problème. On note que trois tarifs sont nécessaires pour régler les problèmes de concurrence entre État et PO au départ du Médoc.

Les tarifs pour les céréales et diverses marchandises allant des coquillages aux choux révèlent une complexité pour le prix de la tk suivant des paliers de distance différents. Un tarif établi pour l'acheminement des choux, choux-fleurs et pommes de terre nouvelles des gares des lignes de Barfleur à Valognes et Montebourg à Paris-Batignolles comprend neuf grilles tarifaires en fonction du poids minimum (50, $100,500 \mathrm{~kg}, 2,5 \mathrm{t}, 4 \mathrm{t}$ ) de la distance, de la marchandise (choux et choux-fleurs ne reçoivent pas le même traitement) et, pour les pommes de terre nouvelles, de l'emploi ou non des trains désignés.

\section{Conclusion}

Au terme de ces analyses, nous enregistrons un seul accord tarifaire entre compagnies secondaires, celui des réseaux landais. Il convient en conclusion de préciser les facteurs qui ont freiné la marche vers une harmonisation tarifaire, bien loin de toute standardisation.

Le simple nombre des compagnies secondaires, plus de 180 en 1912, est un facteur contraignant; on le conçoit en constatant les difficultés pour s'entendre qu'ont les grands réseaux. L'entente entre ces derniers s'impose, leur fonction étant d'assurer les transports au niveau national et international ; leurs réseaux irriguent tout le territoire. L'État exerce sur eux une tutelle unique poussant à une réglementation commune définie dans les cahiers des charges; l'homologation ministérielle des tarifs favorise la cohérence. Les compagnies secondaires 
dépendent des autorités du département dans lequel elles exercent leur activité ; elles desservent des espaces réduits et accordent des avantages tarifaires limités au kilométrage de leurs lignes. Ces chemins de fer assurent les trafics locaux et les acheminements liés aux gares de transit avec les grands réseaux. Cette double fonction leur est commune avec les lignes du plan Freycinet et autres liaisons secondaires exploitées par les grandes compagnies; mais les compagnies secondaires n'ont pas à respecter toutes les règles des grands réseaux, leur indépendance leur permet plus de souplesse dans leur tarification et une adaptation plus immédiate à la demande locale, d'où la grande diversité des tarifs soulignée par l'analyse.

Les grandes compagnies établissent des tarifs communs avec des réseaux secondaires, lorsque c'est indispensable pour atteindre des localités génératrices de trafics appréciables : stations du littoral varois, sucreries des régions betteravières... Leur réticence à la multiplication de ces accords tarifaires s'explique par leur crainte de voir prendre en compte les lignes secondaires dans la définition de l'itinéraire le plus court; la construction de quelques kilomètres de ligne à bon marché pourrait remettre en cause la répartition de certains trafics et raviver la concurrence entre les grands réseaux.

Administrateurs et directeurs varient avec la nature des compagnies. Les administrateurs des grands réseaux sont liés à la haute finance et la grande industrie ; ils sortent parfois des mêmes grandes écoles que leurs cadres de direction. Cette homogénéité favorise des unités de vue; elle n'exclut pas l'esprit de concurrence entre réseaux qui pousse les services commerciaux à multiplier les tarifs spéciaux. Les compagnies secondaires intègrent dans leur conseil d'administration des élus, des notables et des représentants des intérêts économiques locaux. Un polytechnicien ou un ingénieur des Ponts peuvent être à l'origine d'un réseau, ou simplement appelés à le diriger; ils seront entourés d'un personnel moins qualifié et n'auront pas l'influence que leurs camarades d'École exercent par leur nombre au sein des grands réseaux. Ils devront composer avec les représentants des intérêts locaux; ils seront confrontés aux volontés des conseils d'administration. Leur objectif ne sera pas la définition d'une politique commerciale conçue pour de multiples compagnies.

L'idée de voir les dirigeants des compagnies secondaires adopter dans le domaine complexe de la tarification une même classification des marchandises, un même prix de base de la tk et du vk, des barèmes dégressifs identiques suivant le poids et la distance, des avantages tarifaires appliqués non à un trafic précis sur une seule relation mais étendus 
à des mouvements de même nature, en un mot de les voir tendre vers une politique commerciale unifiée n'est pas dans l'esprit du temps. Cela ressort de l'étude des documents tarifaires. Le désir d'une tarification nationale valable pour toutes les compagnies ferroviaires était-il celui de la clientèle ? C'est probable et la revendication n'en serait pas neuve : «En ce qui concerne les marchandises, on nous permettra de formuler le vœu que l'autorité intervienne et agisse peu à peu, afin de ménager la simplification des tarifs actuels, qui sont trop compliqués. L'expéditeur se perd dans les distinctions faites. Cette première simplification préparerait les voies à une autre, non moins désirable, je veux parler de la similitude des taxes sur toutes les lignes. ${ }^{16}{ }^{»}$ Ce texte date de 1856 ! Les compagnies secondaires disparaitront avant toute marche vers une normalisation tarifaire, tandis que la création de la SNCF conduira vers une plus grande homogénéité des tarifs et de leurs conditions d'application sur l'ensemble du réseau exploité.

16- A. Audiganne, «Les chemins de fer et l'ère des grandes exploitations », Revue des deux-mondes, 15 août 1856, p. 743-774, voir p. 770. 


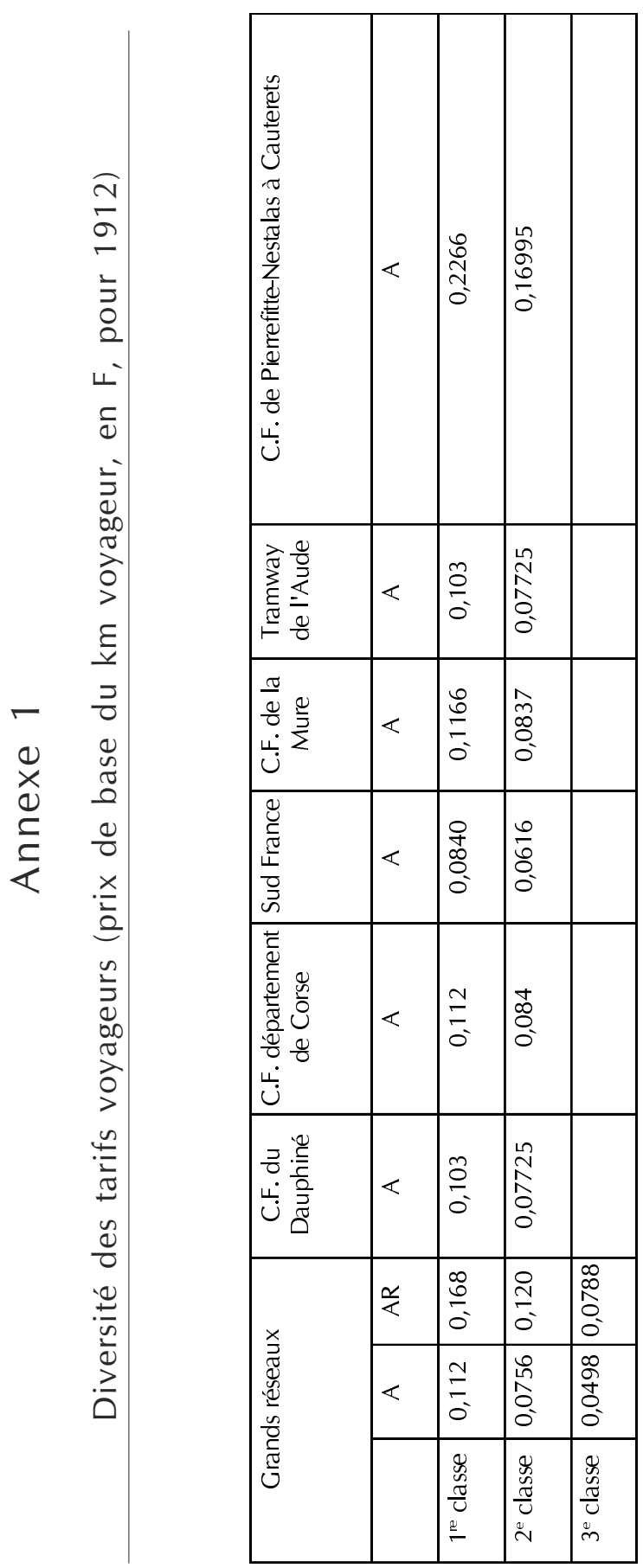




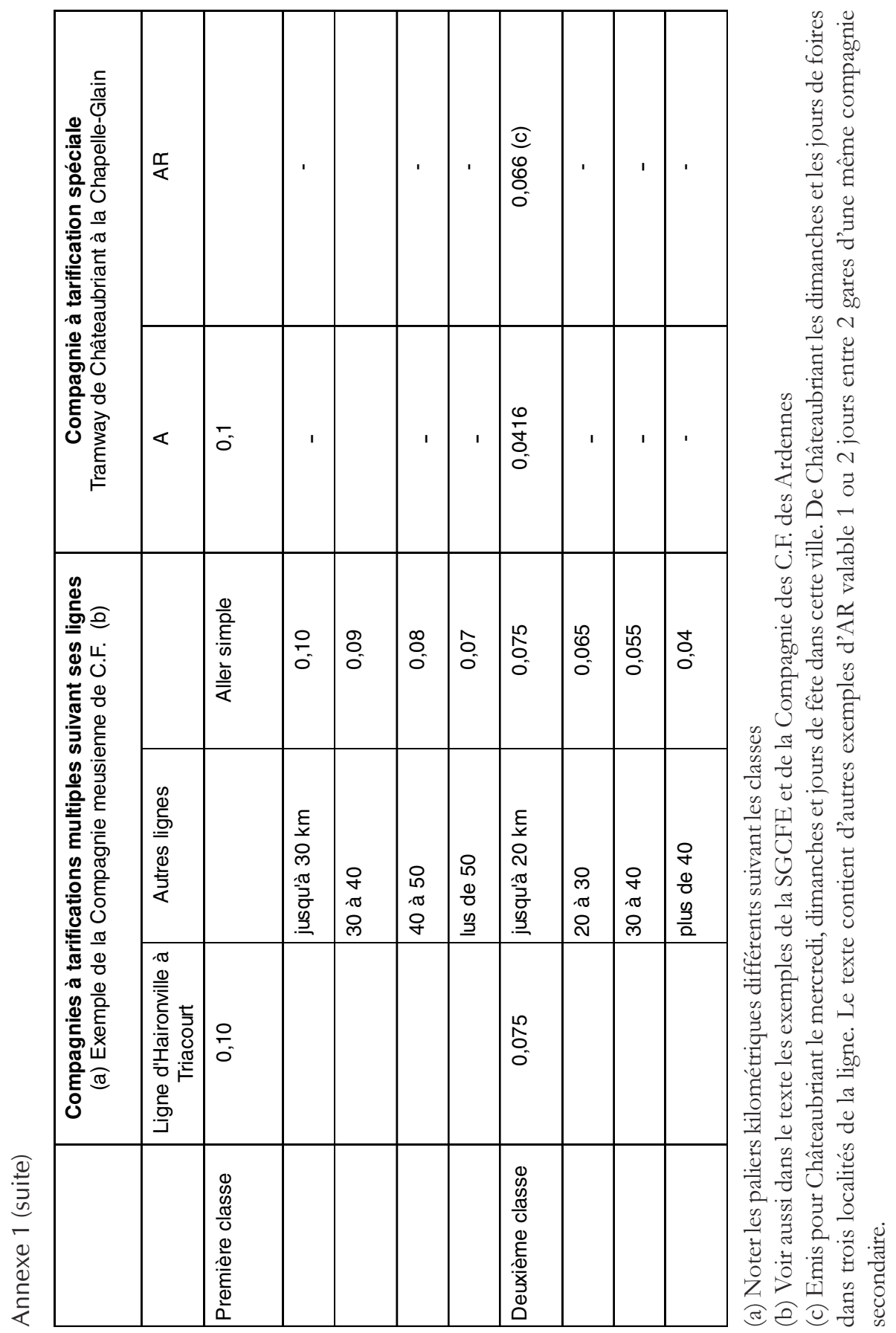




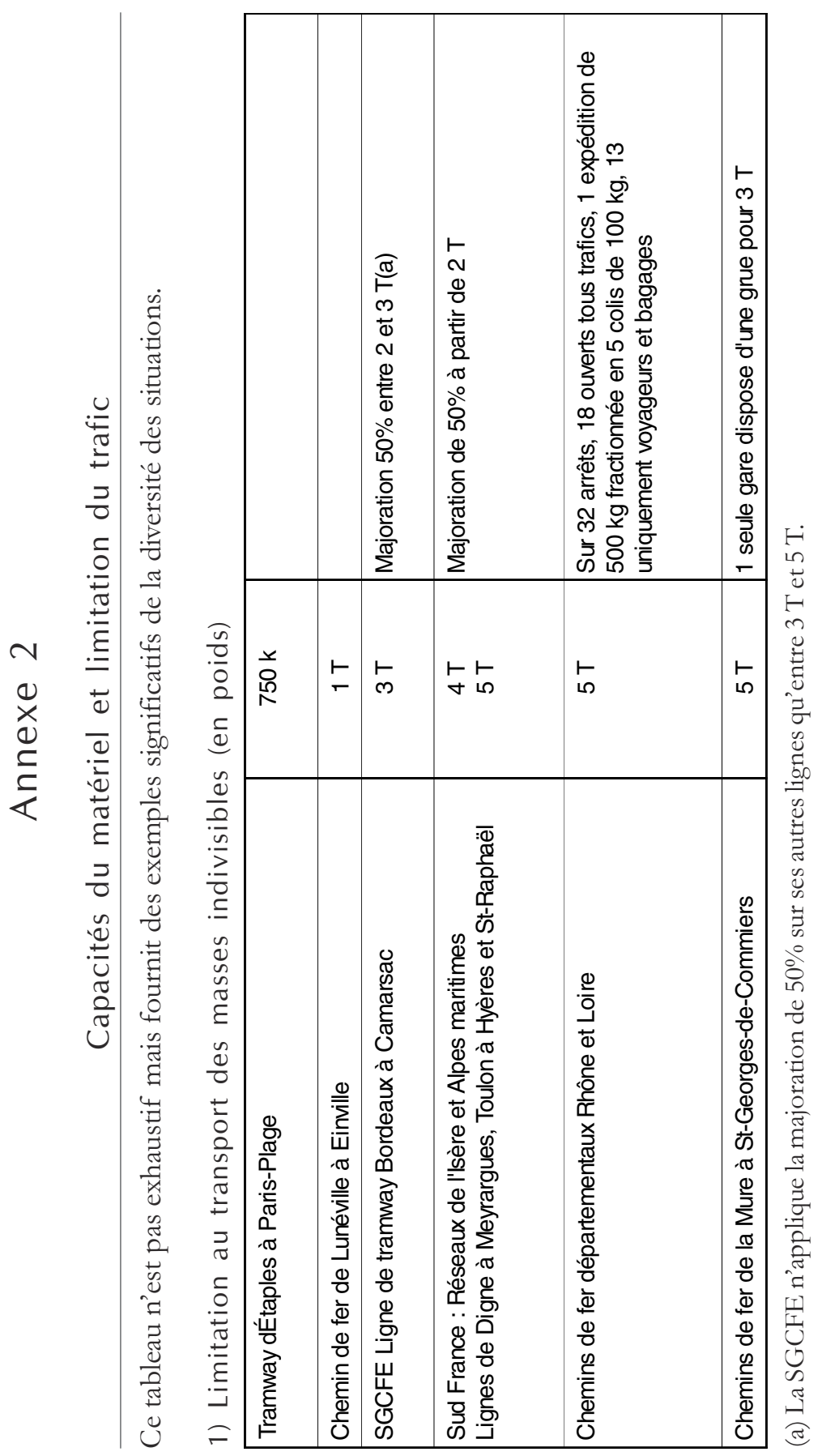




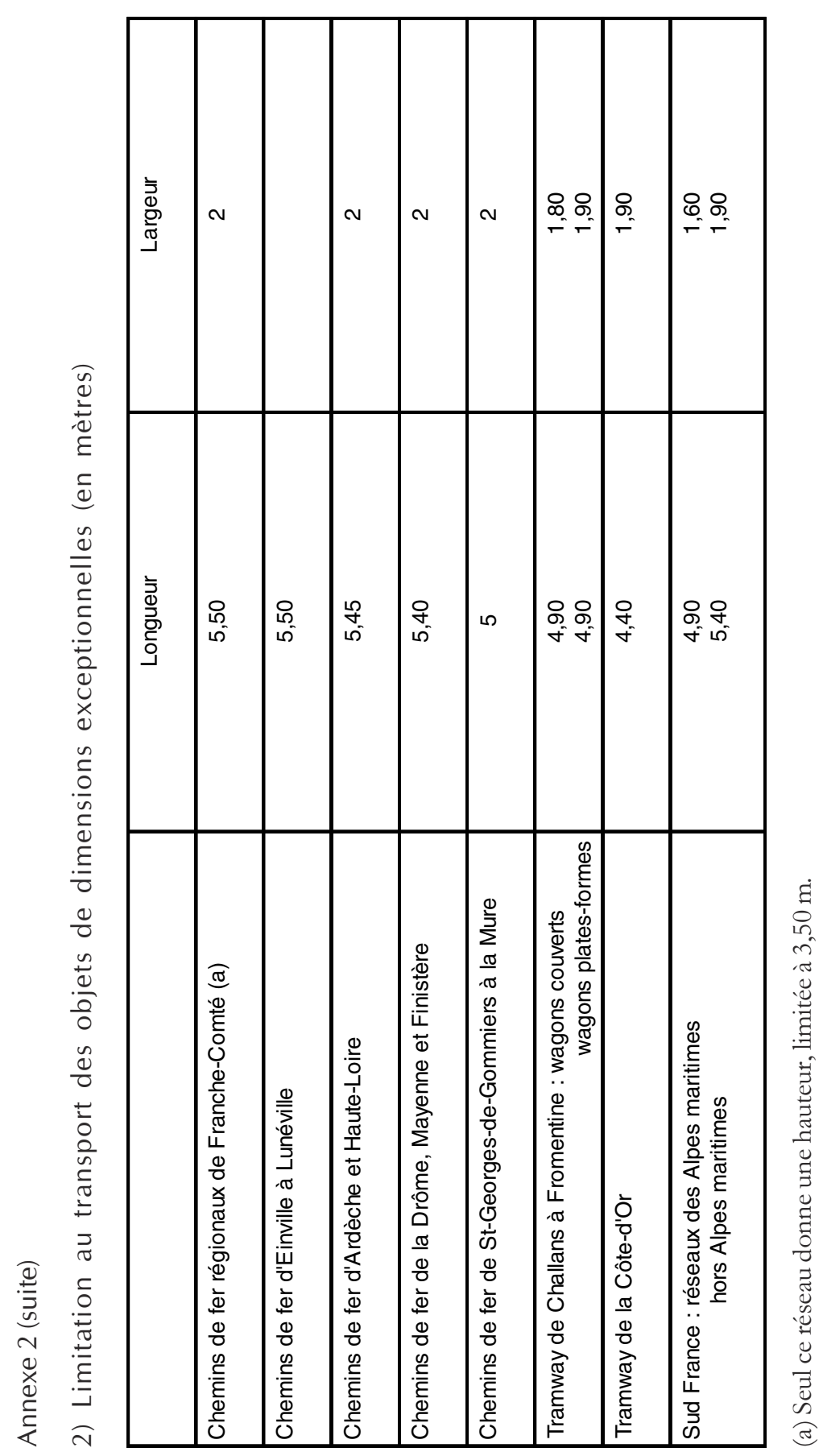




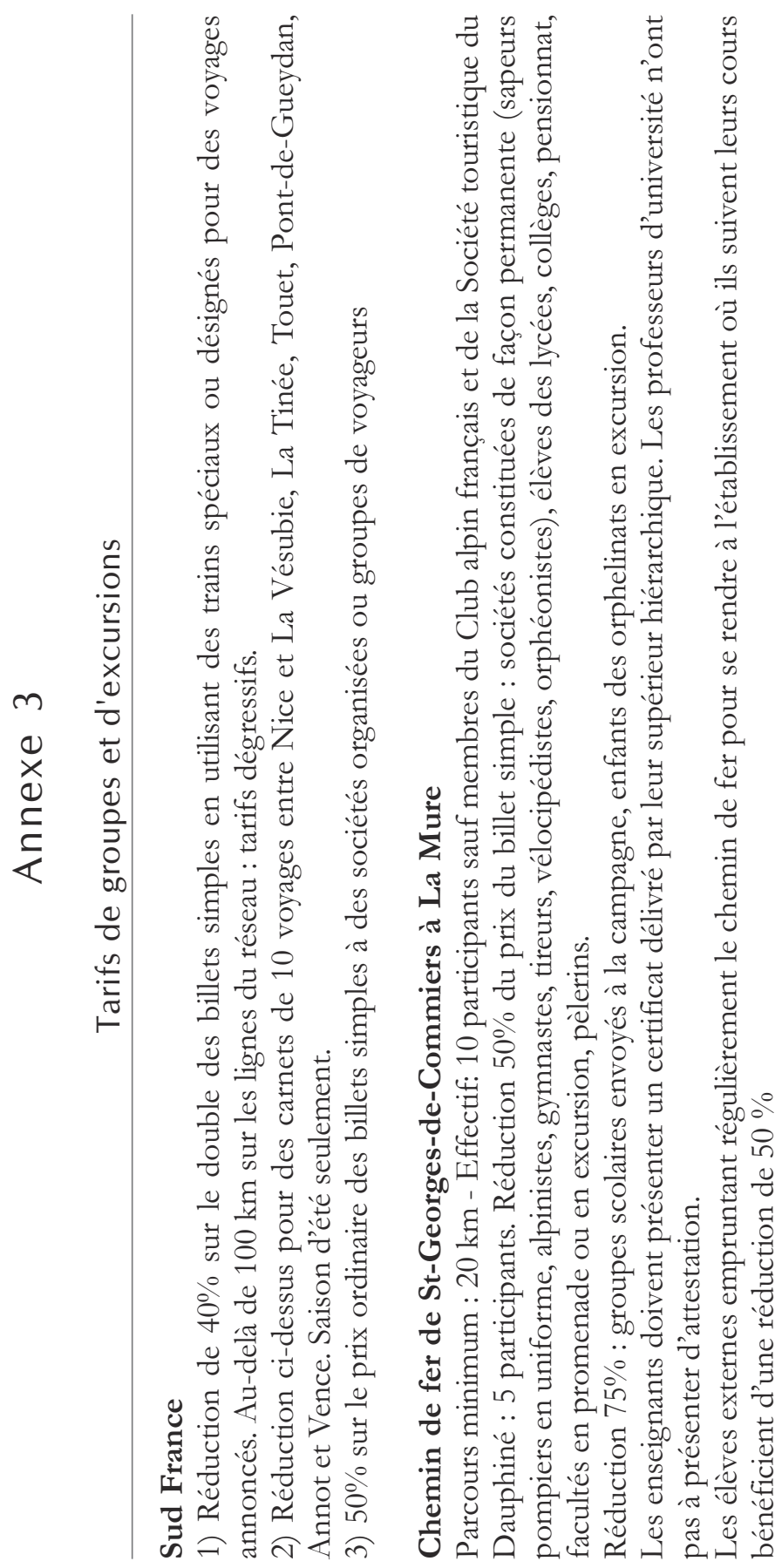




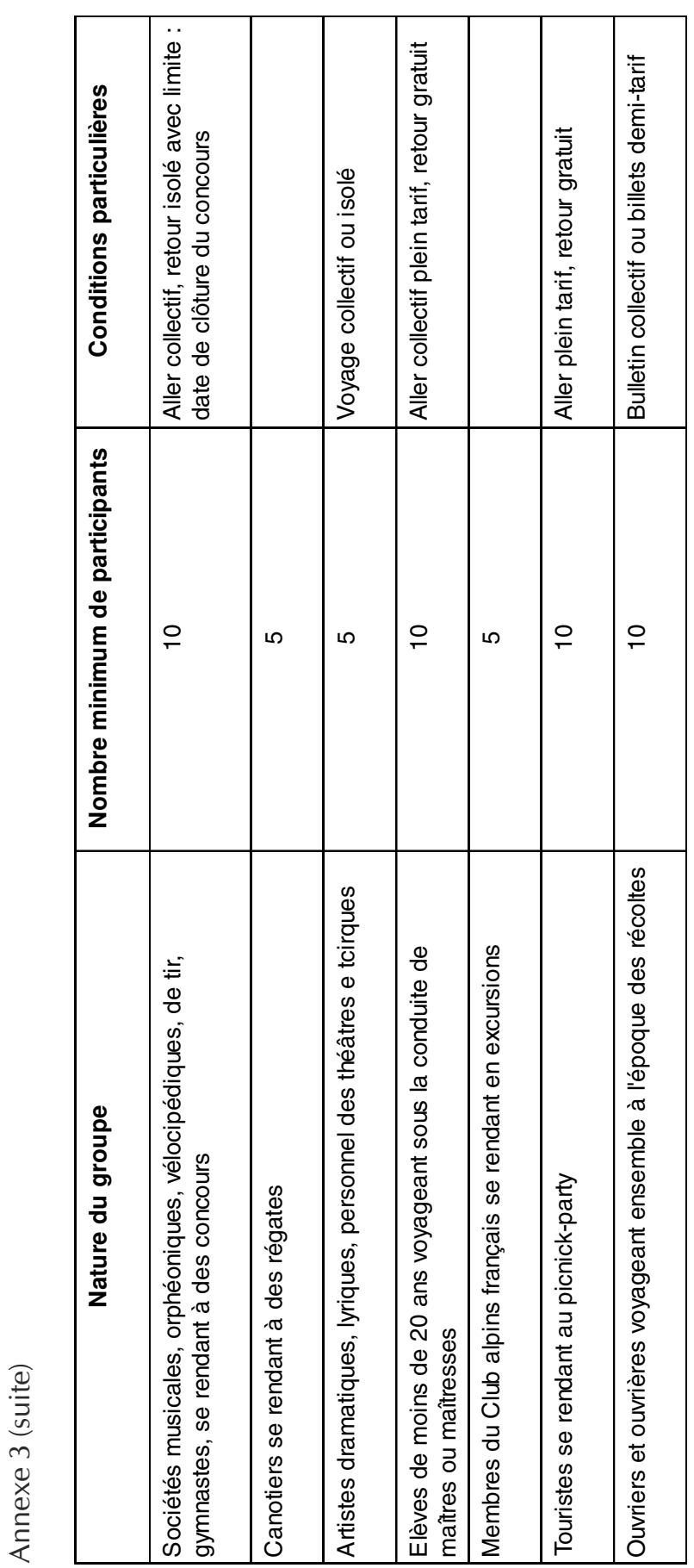




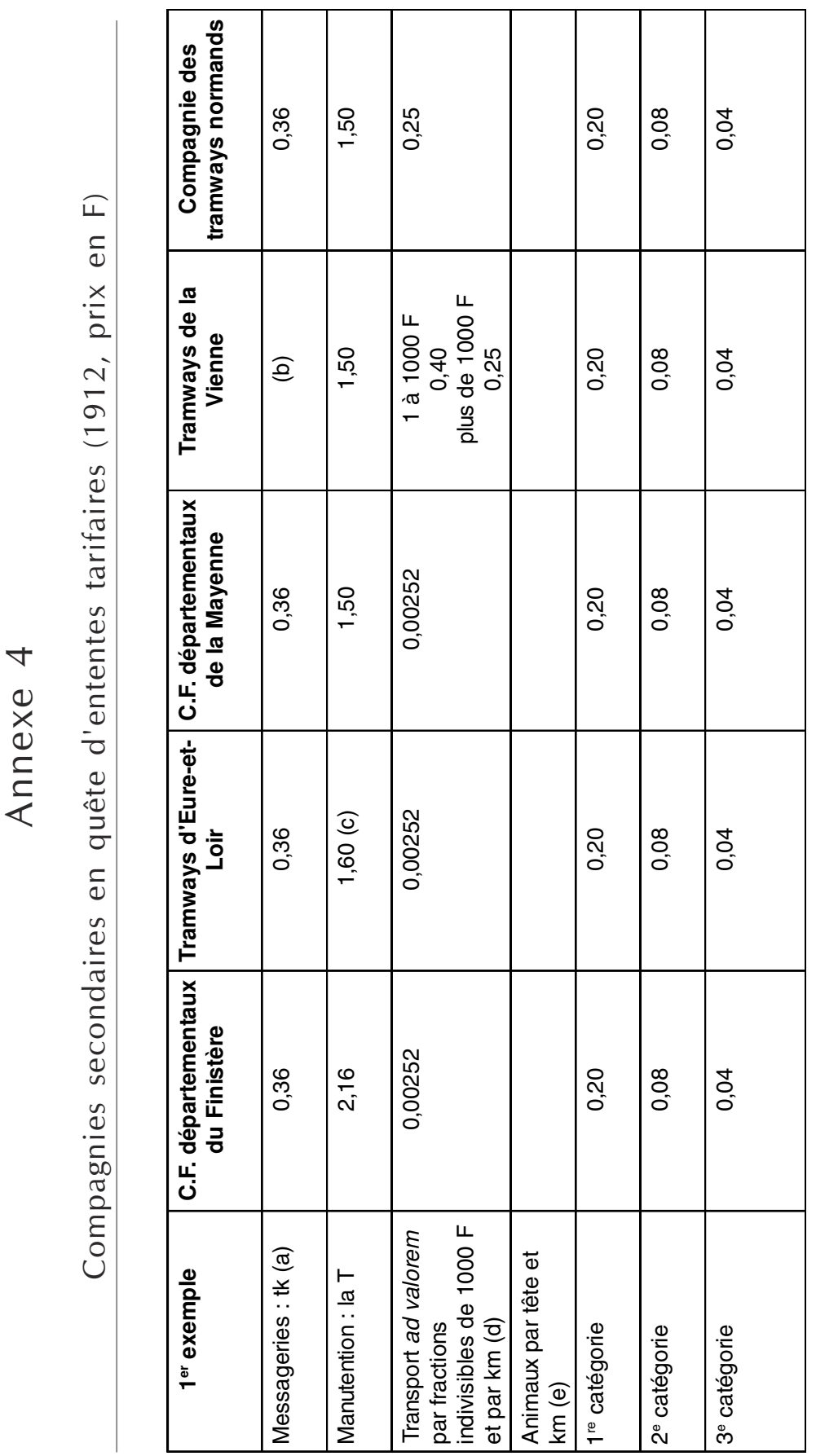




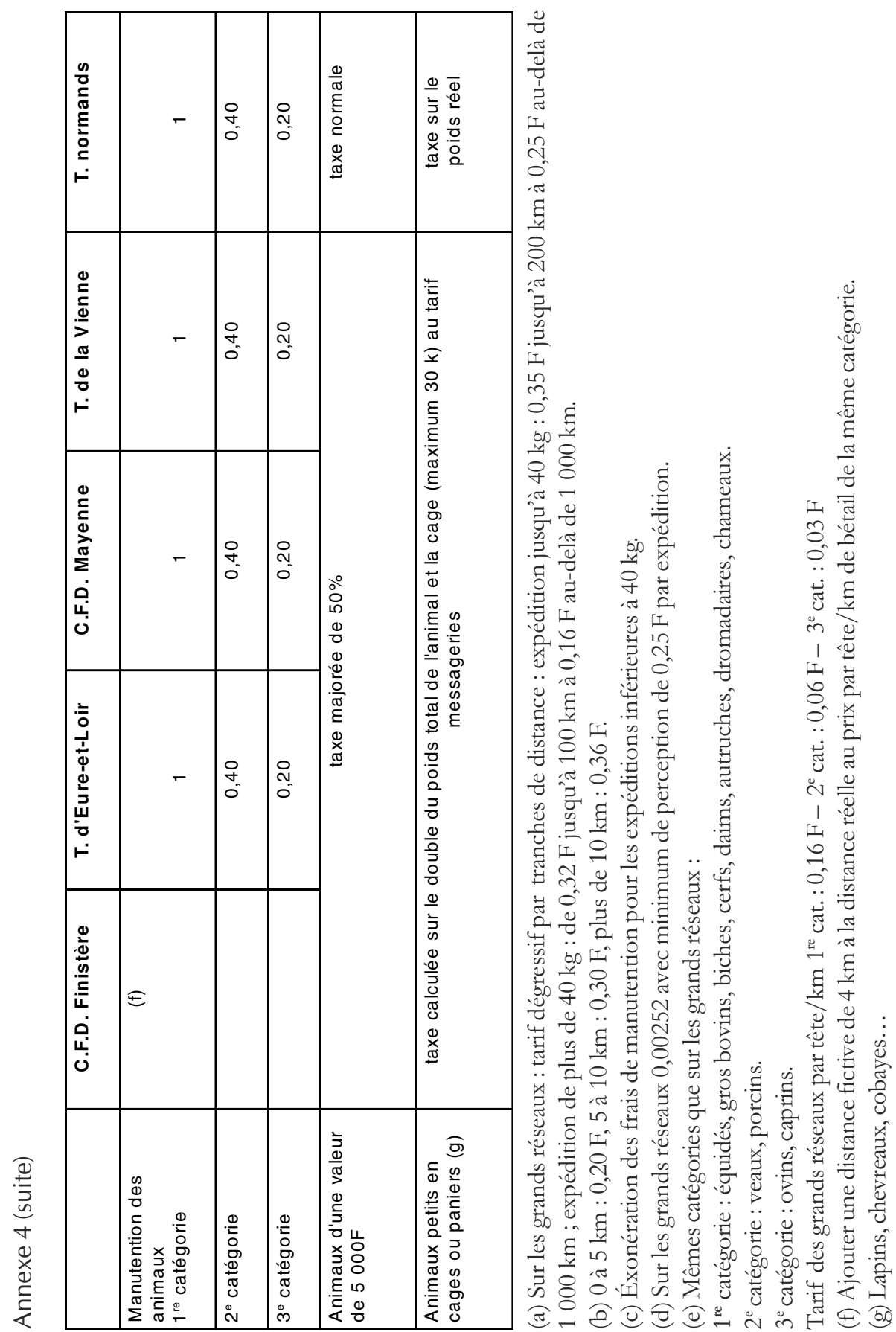




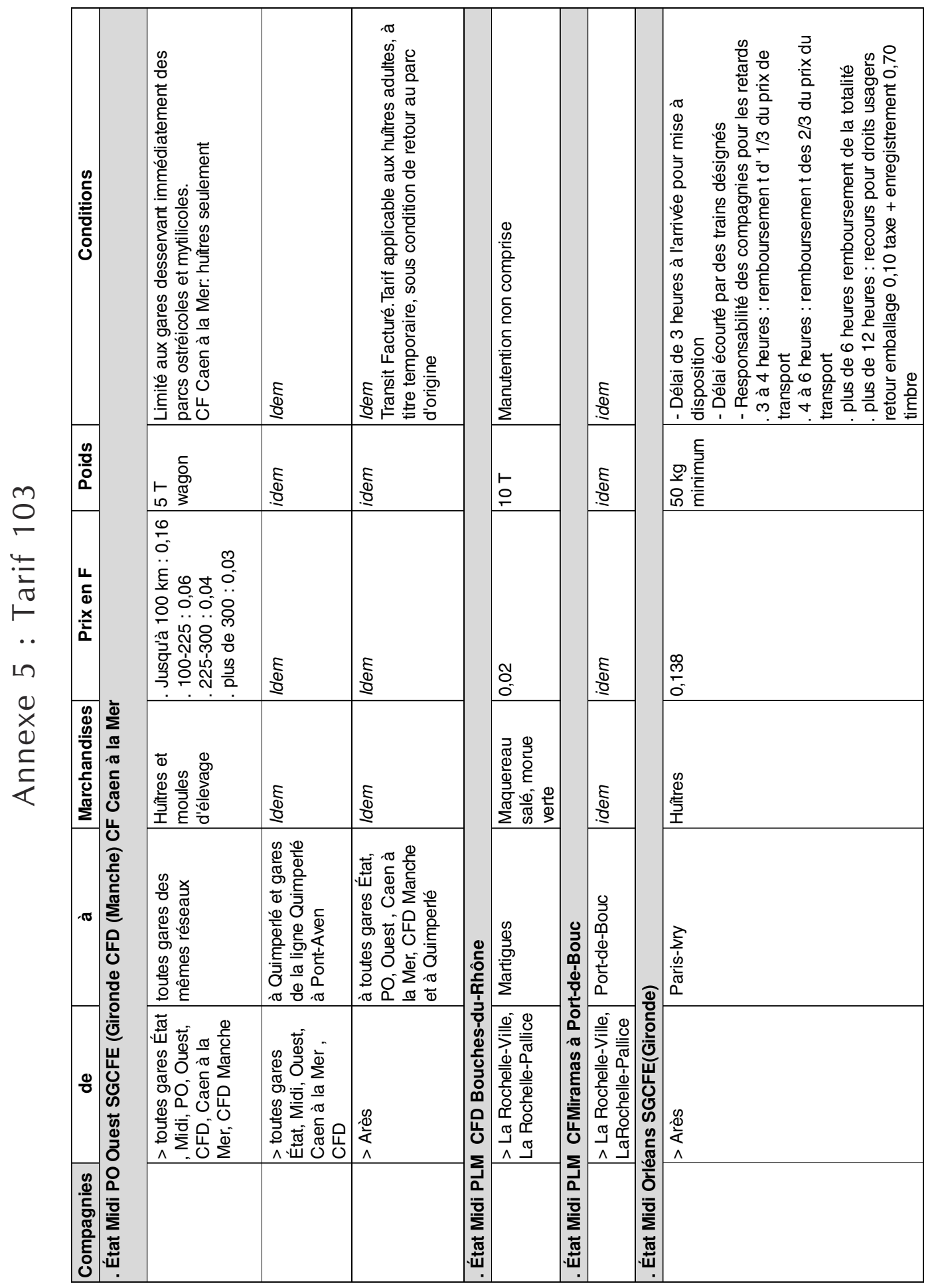




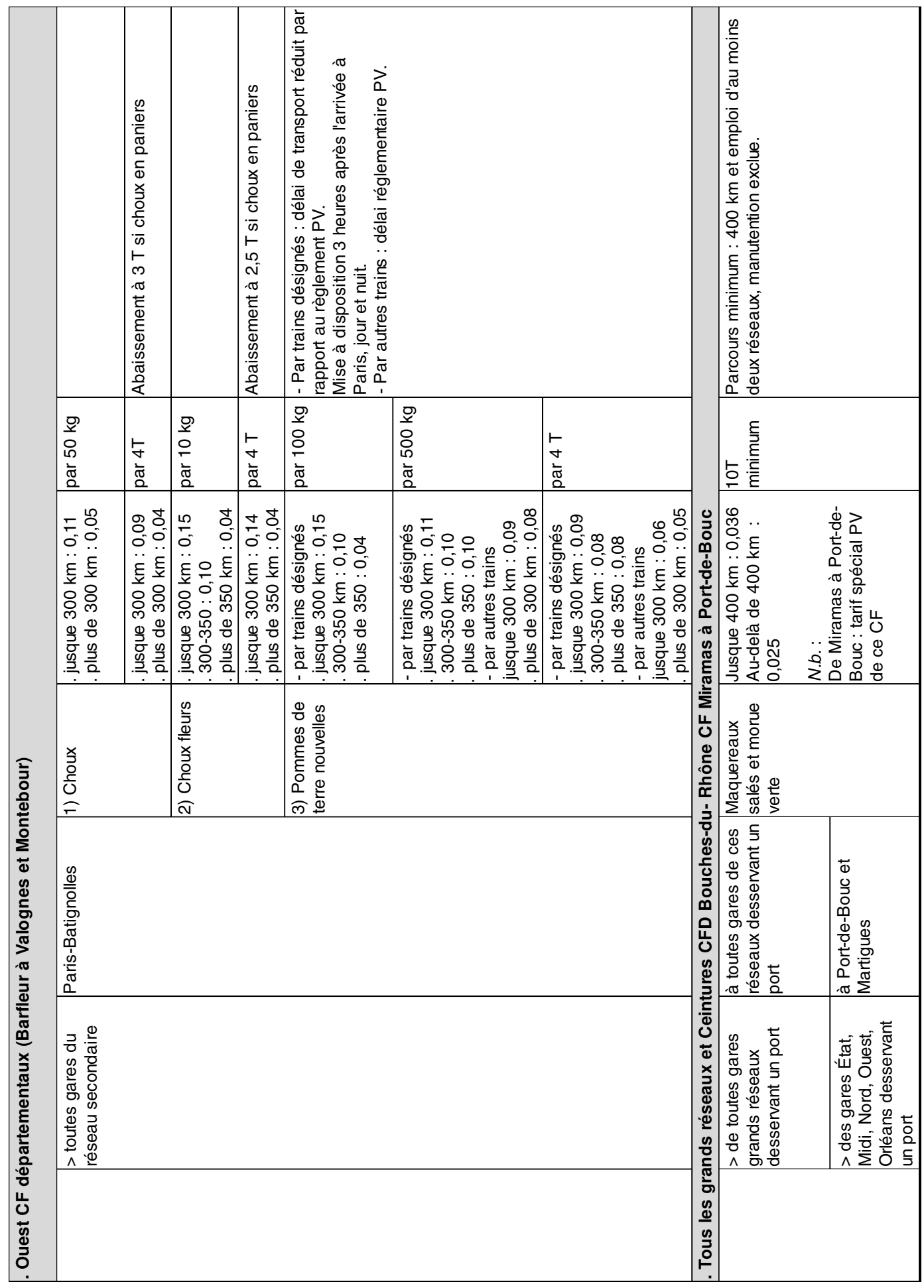

